\title{
Editorial
}

\section{Human Genomics begins ePub ahead of print and a discussion forum Aiming to Bridge Academia with the Pharmaceutical Industry}

I would like to welcome you to Volume 5, Issue 5 of Human Genomics and announce some exciting changes starting with the upcoming Volume 6. As you all know, we have introduced the author-paid optional open access for all article types for a minimal fee of US\$750, just enough to cover the production costs. This makes it rather encouraging for authors to submit their work to Human Genomics, since our journal does not have any page charges, colour figure charges, or supplemental material charges.

The open access, in combination with the $e P u b$ ahead of print, discussed below, will permit immediate public access to both the preliminary accepted version and the copyedited, typeset version published in the online journal. This option is in addition to the open access already provided through the National Institutes of Health's PubMed Central repository; all primary research published in Human Genomics is freely available through PubMed Central six months after publication.

Starting in Volume 6, Human Genomics will have available the e-Pub ahead of print option for all publications. These are citations that publishers submit to PubMed for articles that appear on the publisher's website in advance of the journal release. The reason for the $e P u b$ ahead of print approach is to publish accepted, checked and proofread articles online prior to their appearance in the print version of a journal. Such an article is assigned a digital object identifier (DOI; a character string that serves as a unique identifier for an article and stays with the article even after it has been assigned volume, issue and page numbers), and is indexed by PubMed and other indexing services. As with many other journals, we believe that this is very important, simply because it allows an accepted paper (ePub article) to be cited immediately by its DOI and then associated with its traditional citation after it is published in print. The online access to 'ahead of print' articles will be limited to individual subscribers and to libraries with paid subscriptions.

One of the unique features of Human Genomics is its role as the interface between the pharmaceutical industry and academic research. Our aim is to publish original research papers and review articles relevant to both. To support this feature further, we are introducing a forum, Aiming to Bridge Academia with the Pharmaceutical Industry.

Vasilis Vasiliou Editor of Human Genomics University of Colorado, Denver, CO, USA 